\title{
THE
}

\section{Langevin Analysis of the Diffusion Model for Surface Chemical Reactions}

David L. Freeman

University of Rhode Island, dfreeman@uri.edu

Jimmie D. Doll

Follow this and additional works at: https://digitalcommons.uri.edu/chm_facpubs

Terms of Use

All rights reserved under copyright.

\section{Citation/Publisher Attribution}

Freeman, D. L., \& Doll, J. D. (1983). Langevin Analysis of the Diffusion Model for Surface Chemical

Reactions. J. Chem. Phys., 79(5), 2343-2350. doi: 10.1063/1.446039

Available at: http://dx.doi.org/10.1063/1.446039

This Article is brought to you for free and open access by the Chemistry at DigitalCommons@URI. It has been accepted for inclusion in Chemistry Faculty Publications by an authorized administrator of DigitalCommons@URI. For more information, please contact digitalcommons-group@uri.edu. 


\section{AIP Chemicital Prysics}

\section{Langevin analysis of the diffusion model for surface chemical reactions}

David L. Freeman@f@f and Jimmie D. Doll

Citation: J. Chem. Phys. 79, 2343 (1983); doi: 10.1063/1.446039

View online: http://dx.doi.org/10.1063/1.446039

View Table of Contents: http://jcp.aip.org/resource/1/JCPSA6/v79/i5

Published by the American Institute of Physics.

Additional information on J. Chem. Phys.

Journal Homepage: http://jcp.aip.org/

Journal Information: http://jcp.aip.org/about/about_the_journal

Top downloads: http://jcp.aip.org/features/most_downloaded

Information for Authors: http://jcp.aip.org/authors

\section{ADVERTISEMENT}

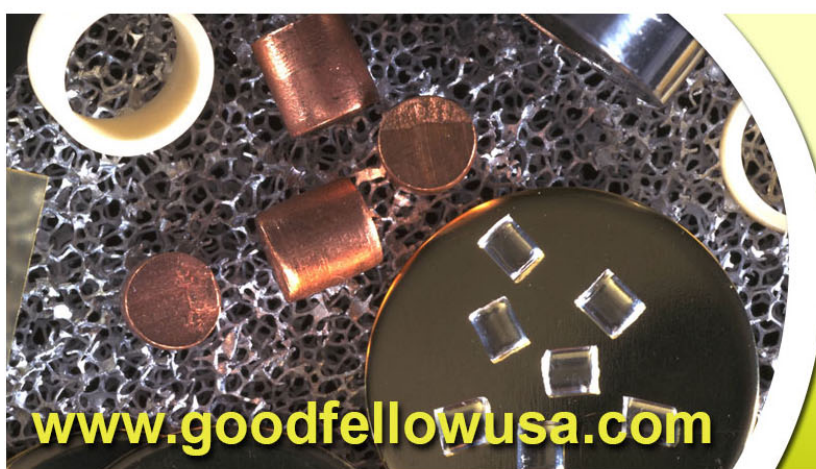




\title{
Langevin analysis of the diffusion model for surface chemical reactions
}

\author{
David L. Freemana) and Jimmie D. Doll \\ University of California, Los Alamos National Laboratory, Chemistry Division, MS G738, Los Alamos, New \\ Mexico 87545 \\ (Received 30 March 1983; accepted 18 May 1983)
}

\begin{abstract}
An analysis is presented of the magnitude of some of the potential sources of error in a recently developed diffusion model of surface chemical reactions. Using single absorber Langevin simulations, comparisons are made between the diffusion equation model and the Fokker-Planck equation for the rates of diffusion controlled surface chemical reactions. The diffusion equation is found to predict rates in good agreement with the Fokker-Planck equation for physical values of the diffusion constant. For unphysically large diffusion constants, the rates predicted by the diffusion equation are found to be in error. By employing multiple absorber Langevin simulations errors in the single absorber approximation used in the diffusion model of surface reactions are examined. The single absorber model is found to be accurate for weakly bound adsorbates. For strongly bound adsorbates rate expressions derived from a two-dimensional model are found to be appropriate. The relative rates of Eley-Rideal and Langmuir-Hinshelwood mechanisms are also studied by multiple absorber Langevin simulations. The ratios of the Eley-Rideal to the Langmuir-Hinshelwood rate is found to be in good agreement with the predictions of the diffusion equation model for physically reasonable diffusion constants. The time dependent solution to the diffusion equation considered in a previous publication is given in an appendix.
\end{abstract}

\section{INTRODUCTION}

In recent years, diffusion constants for the migration of adsorbates on crystal surfaces have become available both from molecular dynamics calculations ${ }^{1-4}$ and a variety of experimental techniques. ${ }^{5}$ The interest in surface diffusion is partially motivated by the fact that diffusion is a primary step in the dynamics of many surface processes. To connect diffusion information with kinetic rate constants in a recent publication ${ }^{6}$ [hereafter referred to as I] we extended the theory for diffusion controlled reactions in solutions ${ }^{7}$ to surface diffusion influenced reactions. In $I$, we analyzed the kinetic implications of the two-dimensional diffusion equation

$$
\frac{\partial W(r, t)}{\partial t}=D \nabla^{2} W(r, t)-\frac{W(r, t)}{\tau}+J,
$$

which was first used for thin film nucleation studies. ${ }^{8}$ In Eq. (1), $W(r, t)$ was the concentration of reactant species at coordinate $r$ and time $t, D$ was the sum of the diffusion constants for reactant molecules, $\tau$ was the lifetime of a reactant molecule before desorption, and $J$ was the number of reactant molecules per unit time per unit area adsorbing on the surface externally from the gas phase. Equation (1) differed from the usual diffusion equation by the addition of terms which allowed reactant molecules to enter or leave the reaction surface.

As pointed out in I, Eq. (1) has two parameters, $J \tau$ and

$\gamma=(D \tau)^{-1 / 2}$,

where $\gamma^{-1}$ can be interpreted as one-half the average

aVisiting Staff Member at the Los Alamos National Laboratory. Permanent address: Department of Chemistry, The University of Rhode Island, Kingston, Rhode Island 02881. distance traveled by a nonreacting molecule before desorption. In terms of the key parameters from Eq. (1), expressions were derived in I for diffusion influenced rate constants, the activation energies for diffusion influenced reactions, and the ratio of the rates of Eley-Rideal processes to Langmuir-Hinshelwood processes. In I, we showed within the model defined by Eq. (1) that the Eley-Rideal rate should be less important than the Langmuir-Hinshelwood rate for many reactions, and we showed the activation energy for Langmuir-Hinshelwood reactions to be bounded by the activation energy for diffusion and the activation energy for recombination. As pointed out in I, the expressions developed for diffusion influenced rate constants in terms of surface diffusion constants are particularly valuable for theoretical study because diffusion constants can be calculated from a portion of the potential energy surface required for full reaction dynamics calculations.

The model developed in I for diffusion influenced rate expressions for surface reactions contains a number of assumptions the effect of which require further analysis. The expressions developed in I are based on the behavior of a two-dimensional isotropic fluid which obeys the diffusion equation. Actual surface reactions occur on a lattice with appreciable activation barriers. The effect of such a lattice may be diffusion constants with spatial anisotropies. In I, isotropic diffusion constants were assumed. The rate constants within the diffusion model are evaluated from expressions for the concentration at an absorption boundary. It is well-known ${ }^{9}$ that the diffusion equation gives inaccurate concentration profiles at such absorbing boundaries, and that a careful treatment requires solutions to the Fokker-Planck equation for the full phase space distribution function. In I by analogy with diffusion influenced reactions in solution the rate expressions were developed from the limiting 
form of a single absorber model. In contrast to solution kinetics the single absorber model may be inappropriate for surface reactions, because the influence of the absorption boundary conditions on the concentration profile is long range in two dimensions.

In the present work, we analyze the magnitude of the errors in I which arise from the use of the diffusion equation within the single absorber approximation. We carry out this analysis by comparing the expressions derived in I with the results of simulations of diffusive processes using the Langevin equation. We show the diffusion equation model to be accurate when the magnitude of the diffusion constant is on the order of those measured and calculated for real physical systems. Only for nonphysically large diffusion constants do we find errors in the use of the diffusion equation to be appreciable.

The organization and contents of the remainder of this paper are as follows. In Sec. II, we compare the rate constants evaluated from the diffusion equation with the results of Langevin simulations within the single absorber model. To justify the parameters used in the simulations, we present the time dependent solution to Eq. (1) which extends the steady-state solution we gave previously. In Sec. II, we compare both rate constants and concentration profiles from the Langevin simulations with the diffusion equation. In Sec. III we compare the results obtained from the single absorber model with multiple absorber simulations. We determine the kinds of parameters for which the results of I are valid, and indicate expressions from I which can be used in other cases. We also calculate the ratio of Eley-Rideal rates to Langmuir-Hinshelwood rates and compare with the expression derived in I for this ratio. In Sec. IV, we summarize our conclusions. We derive the time dependent solution to Eq. (1) in the Appendix and develop the behavior of the solution in a number of limits.

\section{SINGLE ABSORBER LANGEVIN SIMULATIONS}

The migration of adsorbated on crystal surfaces is accurately described by the classical diffusion equation only on time scales which are long compared to the inverse of the effective friction constant. ${ }^{9}$ For time scales on the order of the inverse of the friction constant an accurate treatment of adsorbate migration requires solutions to the Fokker-Planck equation for the full phase space distribution function. For problems with absorbing boundary conditions the inaccuracies in the diffusion equation extend to distances close to the absorbing region at all times. For time scales on the order of the time for molecular motion the FokkerPlanck equation is inaccurate and surface migration must be described by the generalized Langevin equation. 10,11

For diffusion controlled surface reactions it is imagined that the rate limiting step is the time necessary for reactants to diffuse to some critical reaction distance. Once reactants reach a critical distance the reaction is assumed to be very rapid. In $I$, we modeled such diffusion controlled reactions with the diffusion equation given in Eq. (1). The rate constant expres- sions derived in $I$ required the evaluation of the absorption rate at the absorption boundary. This rate was evaluated at steady state. Although the time to reach steady state is long compared to the inverse of the friction constant, the evaluation of the rate at the absorption boundary may be inaccurate, because of deficiencies in the diffusion equation at short distances. To test the errors in the rate derived from the diffusion equation, in this section we present the results of numerical calculations of rates by Langevin simulations. The Langevin simulations give rates equivalent to rates which would be obtained from a solution to the FokkerPlanck equation. The Langevin simulations are carried out with geometries identical to the geometries used in I. Consequently, in this section, errors in the diffusion equation are examined within the single absorber model. Errors introduced by the use of the single absorber approximation will be examined in Sec. III.

To derive expressions for rate constants for diffusion controlled reactions in I, we solved Eq. (1) at steady state subject to the boundary conditions

$$
W_{D}\left(R_{A}\right)=0
$$

and

$$
W_{D}\left(R_{B}\right)=C_{0},
$$

where $W_{D}(r)$ is the steady state solution to Eq. (1), $R_{A}$ is a critical distance within which we assume all diffusing absorbates to react with unit probability, and $R_{B}$ is an outer radius which provides a source of reactants at the initial concentration $C_{0}$. The solution to Eq. (1) at steady state subject to the boundary conditions given in Eqs. (3) and (4) was found to be

$$
W_{D}(r)=J \tau\left[1+A K_{0}(\gamma r)+B I_{0}(\gamma r)\right] \text {, }
$$

where $I_{n}(x)$ and $K_{n}(x)$ are modified Bessel functions of the first and second kind of order $n$, and $A$ and $B$ are coefficients whose detailed expressions are given in I [Eqs. (49) and (50)]. The rate constants were extracted from Eq. (5) from the relation

$$
k_{D}=\frac{2 \pi R_{A} D}{C_{0}}\left(\frac{d W_{D}}{d r}\right)_{r=R_{A}} .
$$

In I, Eq. (6) was evaluated in a number of limits of which

$$
\lim _{R_{B^{-\infty}}} k_{D}=k_{D_{\infty}}=2 \pi R_{A} D \frac{\gamma K_{1}\left(\gamma R_{A}\right)}{K_{0}\left(\gamma R_{A}\right)}
$$

and

$$
\lim _{\gamma \rightarrow 0} k_{D}=k_{D_{0}}=\frac{2 \pi D}{\ln \left(R_{B} / R_{A}\right)}
$$

are important to the present discussion. For finite $R_{B}$ and $\gamma$ the rate constant from Eqs. (5) and (6) is

$$
k_{D}=2 \pi R_{A} D \gamma\left[B I_{1}\left(\gamma R_{A}\right)-A K_{1}\left(\gamma R_{A}\right)\right] .
$$

In Eqs. (6), (7), and (9) we have taken $C_{0}=J \tau$ for convenience.

Because a particle moving in a viscous medium will only display diffusive behavior at times long compared with the inverse of the friction constant it is well known that the diffusion equation provides a poor description 
of stochastic motion at short times and short distances. ${ }^{\circ}$ To describe the distribution of particles near the adsorber at radius $R_{A}$ for times long relative to the time scale of molecular motions it is necessary to solve the Fokker-Planck equation for the full phase space distribution function. When particles are allowed to flow into and out of the surface with the parameters of Eq. (1) the Fokker-Planck equation is

$$
\begin{aligned}
\frac{\partial f}{\partial t}+u \cdot \nabla_{r} f & =\frac{3}{\beta m D} f+\frac{1}{\beta m D} u \cdot \nabla_{u} f \\
& +\frac{1}{\beta^{2} m^{2} D} \nabla_{u}^{2} f-\frac{f}{\tau}+J\left(\frac{m \beta}{2 \pi}\right) \exp \left(-\beta m u^{2} / 2\right) .
\end{aligned}
$$

In Eq. (10), $f(\mathrm{r}, \mathrm{u}, t)$ is the phase space distribution function, $m$ is the mass of the particles, $u$ is the particle velocity, and $\beta=\left(1 / k_{B} T\right), k_{B}$ being the Boltzmann constant, and $T$ being the absolute temperature. Direct solutions to Eq. (10) subject to absorbing boundary conditions are difficult to obtain. ${ }^{12}$ To compare the rate constants and concentrations profiles obtained from Eq. (1) with Eq. (10) we found it more convenient to solve the equivalent Langevin equation

$$
\frac{d \mathrm{u}}{d t}=-\lambda \mathrm{u}+\mathrm{R}(t) \text {. }
$$

In Eq. (11), $\lambda$ is the friction constant related to the diffusion constant by

$$
\lambda=\frac{1}{\beta m D}
$$

and $\mathbf{R}(t)$ is a random force. To solve Eq. (11) under conditions appropriate for the present study we used the geometry shown in Fig. 1. At time $t=0$, particles were scattered over the entire region at random so that the average concentration was $C_{0}$ and the velocities we thermalized to temperature $T$. For each integration, time step particles were added to region $C$ to maintain a constant outer concentrations of $C_{0}$. Reflecting boundary conditions were applied to region $\mathrm{C}$ at the square walls. Particles were allowed to pass freely between regions $B$ and $C$. Particles entering region $A$ were removed and counted as absorbed. To account for a desorption mechanism particles in region $B$ were removed in each time step with probability $\Delta t / \tau$, where $\Delta t$ was the length of a time step. Particles were adsorbed onto region $B$ according to a Poisson distribution with an average number of particles $J \pi\left(R_{B}^{2}-R_{A}^{2}\right) \Delta t$ added in each time step. The Langevin equation was solved by methods implicit in Eq. (240) of Ref. 9 using Gaussian random noise for the integrated random force.

To understand the parameters used in the Langevin simulations it is useful to consider the solution to the time dependent diffusion equation [Eq. (1)]

$W(r, t)=W_{D}(r)+\exp (-t / \tau) \sum_{n=1}^{\infty} c_{n} V_{0}\left(\alpha_{n} r\right) \exp \left(-\alpha_{n}^{2} D t\right)$.

The coefficients $c_{n}$ and $\alpha_{n}$, and the function $V_{0}\left(\alpha_{n} r\right)$ used in Eq. (13) are defined in the Appendix along with a derivation of the equation. Because rate constants

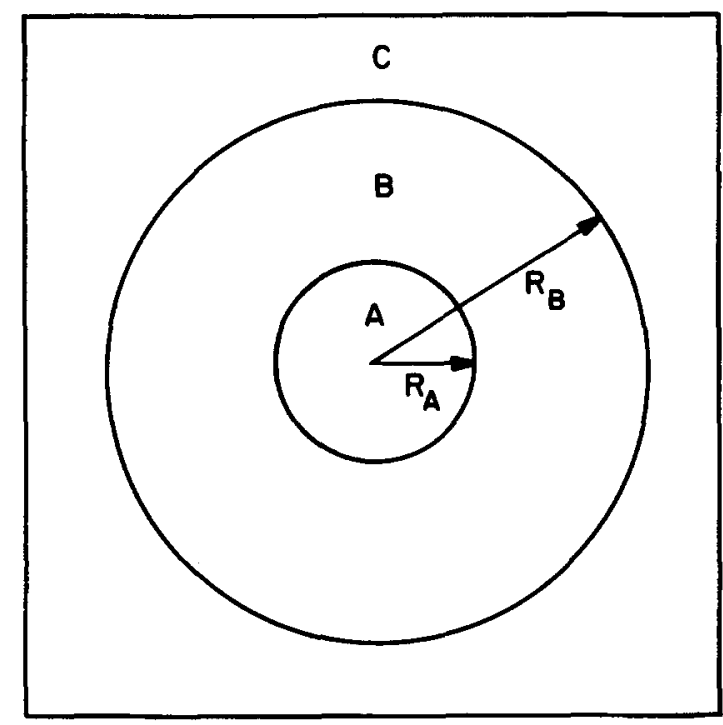

FIG. 1. The geometry used in the single absorber simulations.

are defined at steady state we wish to solve the Langevin equation at steady state. We see that one parameter which will govern the rate of decay of the transient part of Eq. (13) is $\tau^{-1}$. For small $\tau$ the transient solution will decay quickly and a comparison with the steady state solutions will be meaningful. Consequently, in the Langevin simulations we chose very short lifetimes to desorption so that steady state comparisons could be made.

The spatial concentrations used initially in the Langevin simulations are the same as the boundary conditions used to solve Eq. (1); namely,

$$
\begin{aligned}
& W(r, 0)=C_{0}, \quad R_{A}<r \leq R_{B}, \\
& W\left(R_{A}, t\right)=0, \quad t>0, \\
& W\left(R_{B}, t\right)=C_{0}, \quad t>0 .
\end{aligned}
$$

By construction the diffusion equation and FokkerPlanck equation concentration profiles will agree exactly at $t=0$. The principal discrepancies between the Fokker-Planck and diffusion equation concentration profiles will occur at long times for distances $r$ near $R_{A}$. To observe the discrepancies between the FokkerPlanck and diffusion equation concentration profiles, Langevin simulations were carried out with $R_{A}=20$, $R_{B}=50, C_{0}=0.04, T=300 \mathrm{~K}, m=29166$ [that of an oxygen atom], $D=10^{-3}, J=0.04 \times 10^{-5}$, and $\tau=10^{5}$; all numbers expressed in a. $u$. The concentration profile evaluated from a 100 trajectory study at $t=500000$ a. u. is shown in Fig. 2. In Fig. 2, the solid line represents the concentration profile evaluated from the Langevin simulations and the dashed line gives the concentration evaluated with Eq. (5). The evaluated points are given for the Langevin case. The concentration from the diffusion equation underestimates the Fokker-Planck concentration profile in agreement with recent onedimensional studies of Harris. ${ }^{12}$ The concentration falls to zero far more abruptly in the Fokker-Planck case than the diffusion case. This does not necessarily imply a larger absorption rate because $\mathrm{Eq} .(6)$ is only valid for diffusion equation solutions. 


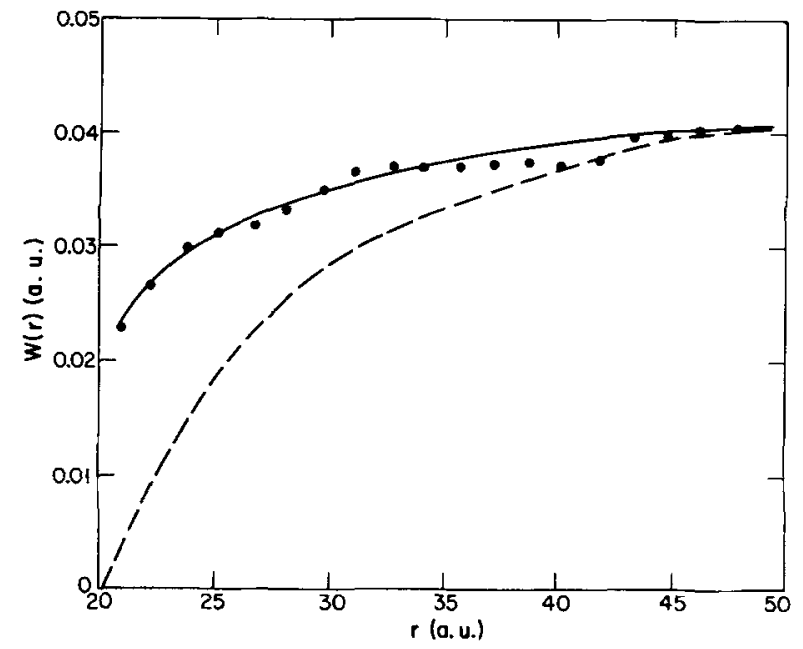

FIG. 2. The concentration of reactants as a function of distance from the absorber. The solid line is the Langevin result and the dashed line is the diffusion equation result.

Absorption rate constants were calculated from the Langevin simulations by evaluating the number of particles entering region $A$ of Fig. 1 as a function of time. An example of a graphical analysis of the rate data is given in Fig. 3. In Fig. $3, R_{A}=2, R_{B}=50, C_{0}=0.01, T$ $T=300 \mathrm{~K}, D=10^{-4}, m=29166, J=10^{-6}$, and $\tau=10^{5}$, all in a. $u$. The Langevin simulations consisted of 100 trajectories. The number of particles absorbed $N$ increases linearly in time from $5 \times 10^{4}$ to $25 \times 10^{4}$ a. u. in time, implying that a steady-state rate of absorption has been attained for $\tau=10^{5}$ a. u. The rate is ob-

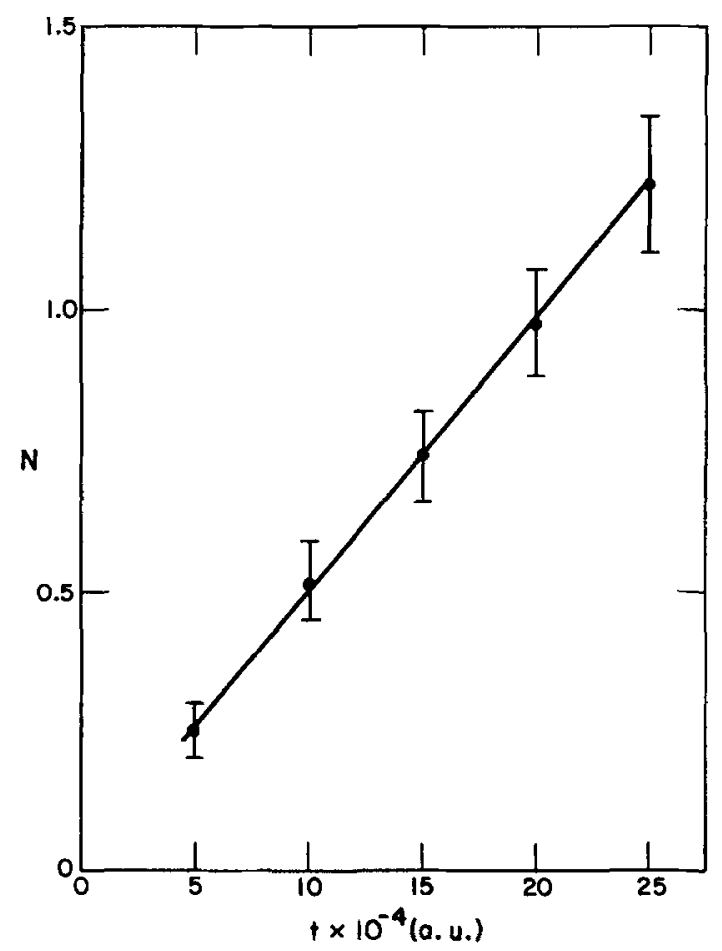

FIG. 3. The number of absorbed particles as a function of time from single absorber Langevin simulations.
TABLE I. Rate constants as a function of diffusion constant.

\begin{tabular}{cccc}
\hline \hline$D^{2}$ & $k_{D}{ }^{2}$ & $k_{D \infty^{2}}$ & $k_{L}{ }^{2}$ \\
\hline $1 \times 10^{-2}$ & $2.25 \times 10^{-2}$ & $2.17 \times 10^{-2}$ & $8.99 \times 10^{-4}$ \\
$1 \times 10^{-3}$ & $3.42 \times 10^{-3}$ & $3.42 \times 10^{-3}$ & $7.11 \times 10^{-4}$ \\
$5 \times 10^{-4}$ & $2.03 \times 10^{-3}$ & $2.03 \times 10^{-3}$ & $7.02 \times 10^{-4}$ \\
$2 \times 10^{-4}$ & $1.05 \times 10^{-3}$ & $1.05 \times 10^{-3}$ & $5.74 \times 10^{-4}$ \\
$1 \times 10^{-4}$ & $6.54 \times 10^{-4}$ & $6.54 \times 10^{-4}$ & $4.94 \times 10^{-4}$ \\
$5 \times 10^{-5}$ & $4.14 \times 10^{-4}$ & $4.14 \times 10^{-4}$ & $3.04 \times 10^{-4}$ \\
$1 \times 10^{-5}$ & $1.54 \times 10^{-4}$ & $1.54 \times 10^{-4}$ & $1.98 \times 10^{-4}$ \\
\hline
\end{tabular}

a.u.

tained from the slope of Fig. 3 and the rate constant is defined as this rate per unit concentration. Rate constants from Langevin simulations $k_{L}$ are compared with $k_{D}[\mathrm{Eq} .(6)]$ and $k_{D \infty}[\mathrm{Eq} .(7)]$ in Table I. The values used for $R_{a}, R_{B}, C_{0}, m, J, T$, and $\tau$ are the same in Table $I$ as in Fig. 3. There is complete agreement between $k_{D}$ and $k_{D \infty}$ for all values of $D$ less than $10^{-2}$. For $D=10^{-2}, \gamma^{-1}=31.62$ so that the concentration profile has not leveled off at $R_{B}=50$. Consequently, the behavior at $R_{B}=50$ is somewhat different than the limiting case of infinite $R_{B}$. For $D=10^{-3}$ or less $\gamma^{-1} \leq 10$ so that the steady-state solution to Eq. (1) at $R_{B}=50$ is the same as at infinite $R_{B}$. The rate constants evaluated from the diffusion model are nearly linear in $D$ as can be understood qualitatively from Eq. (7). This linear behavior is not observed from the Langevin simulations. For large values of $D, k_{L}$ is nearly $D$ independent. The near $D$ independence occurs for large $D$ because the small friction constant leads to nearly free translational motion within the geometry of the calculation. Indeed,

$$
\lim _{D \rightarrow \infty} k_{L}=\text { const , }
$$

in contrast to the diffusion equation behavior. To make the comparison of $k_{D}$ and $k_{L}$ clearer we plot $-\log _{10} D$ against $k_{D} / k_{L}$ in Fig. 4. The ratio is largest for large

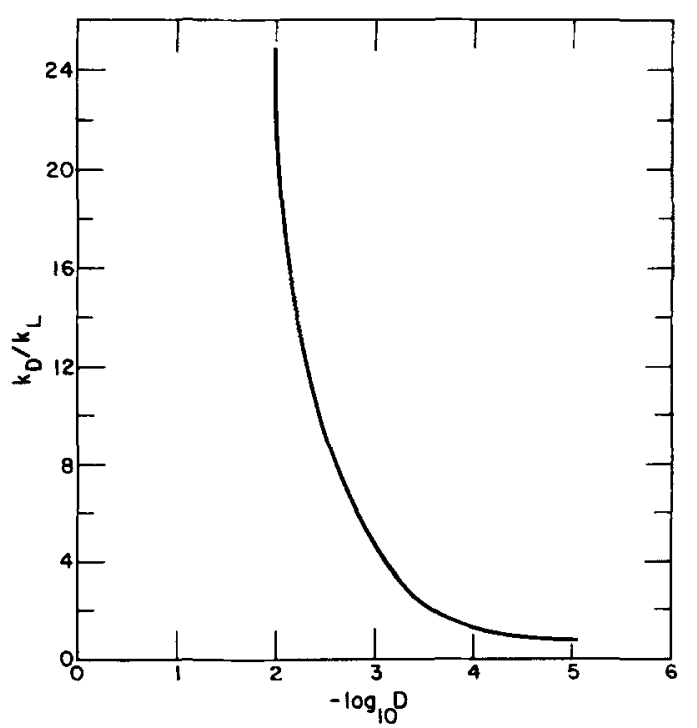

FIG. 4. The ratio of the diffusion equation rate constant to the Fokker-Planck rate constant as a function of the diffusion constant. 


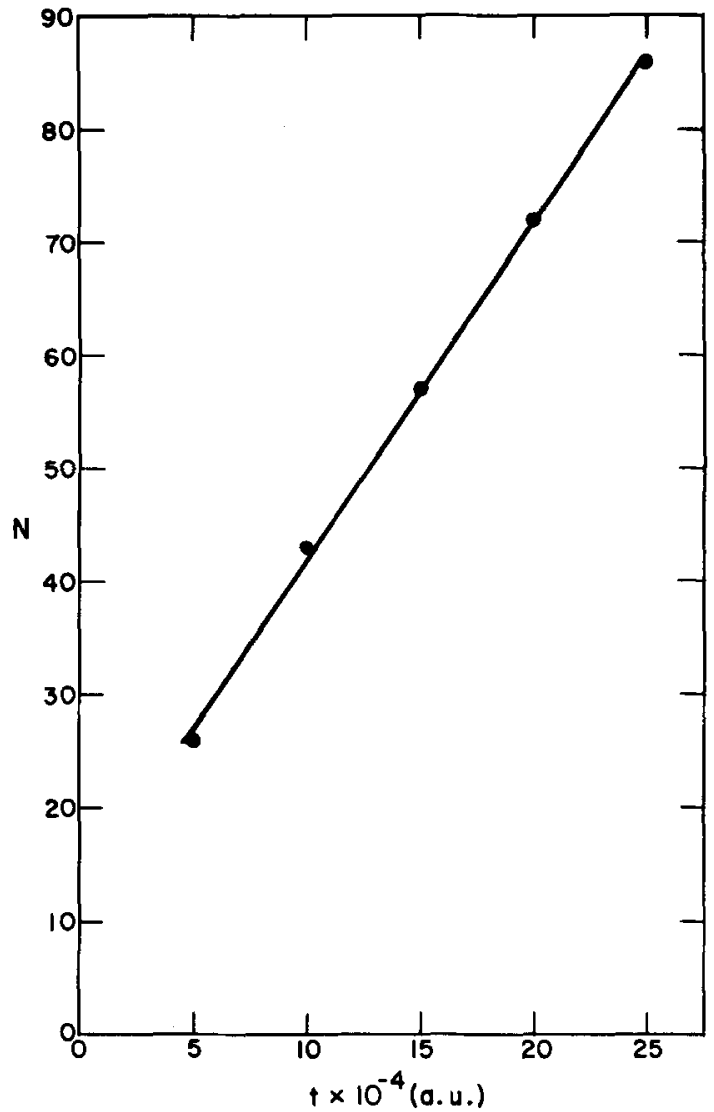

FIG. 5. The number of reacted particles as a function of time from multiple absorber Langevin simulations.

values of $D$ because of Eq. (17). As $D$ becomes small the ratio appears to approach unity. Diffusion constants determined for physical systems tend to be on the order of $10^{-4}$ or $10^{-5}$ a. u. Consequently, the expressions for diffusion influenced rate constants developed in I can be expected to be sufficiently accurate for kinetic analysis of diffusion constant information. We can expect that basing a rate theory on the diffusion equation rather than the Fokker-Planck equation will be accurate for physical diffusion constants.

\section{MULTIPLE ABSORBER SIMULATIONS}

In solution kinetics, rate constants for diffusion controlled reactions are extacted from diffusion constants in the limit that $R_{B}$ is taken to infinity. The infinite $R_{B}$ limit is physically appropriate for reactions in solution, because such reactions are always dilute owing to the presence of a solvent. In addition, the influence of the outer boundary at $R_{B}$ is weak for solution reactions in contrast to the case of diffusion in two dimensions where the outer boundary has a strong influence on the concentration profile and calculated rate constants (see I, Sec. III A). The infinite $R_{B}$ limit is the essence of the single absorber model discussed in Sec. II.

To help assess the validity of the single absorber approximation (or infinite $R_{B}$ limit), we carried out a series of multiple absorber Langevin simulations to compare with the diffusion controlled reaction rate constants evaluated in I and in Sec. II of this work. At time $t=0$, we dispersed 100 particles at random with thermalized velocities on a square two-dimensional surface of dimension $100 \times 100$ a. u. We propagated the motions of the particles with the Langevin equation. In each time step, we remove and counted as reacted any two particles whose centers were separated by a distance $R_{A}$. To simulate the absorption and desorption processes discussed in Sec. II particles were desorbed with probability $\Delta t / \tau$ in each time step of length $\Delta t$, and an average of $10^{4} \mathrm{~J} \Delta t$ particles were added to the surface in each time step. To approximate an extended system periodic boundary conditions were imposed at the walls of the surface.

In each simulation the parameters were taken to be $R_{A}=2, C_{0}=0.01, T=300 \mathrm{~K}, m=29166, J=10^{-6}$, and $\tau=10^{5}$, all in a. u. For each diffusion constant, we ran 100 trajectories except for $D=10^{-5}$ a. u., where ten trajectories were run. The decrease in the number of trajectories was necessary for small diffusion constants, because the computer time became prohibitive. For each calculation the number of reacted particles was measured as a function of time from $t=5 \times 10^{4}$ to $t=25 \times 10^{4}$ a. u. The data were then plotted an example of which is shown in Fig. $5\left(D=10^{-4}\right.$ a. u. $)$. Unlike the single absorber simulations (Fig. 3) no error bars are shown in Fig. 5, because the errors are smaller than the resolution of the graph. The slope of the graph of the number of reacted particles $N$ as a function of time gives the reaction rate. To extract a rate constant from the rate it is necessary to determine the steady-state concentration of particles on the surface which in general will differ either from $C_{0}$ or $J \tau$. The steady state concentration was determined by monitoring the number of remaining surface particles at the end of each time step.

In Table II, we present the final concentration $C_{f}$ the multiabsorber rate constant $k_{M A}$ along with $k_{D}$ and $k_{L}$ for each calculated value of $D$. The values of $D$ used to evaluate $k_{D}$ and $k_{L}$ were twice that used for $k_{M A}$, because the total diffusion constant for both reactants are required in the single absorber model. It is important to recognize that a rigorous comparison between $k_{H A}$ and $k_{D}$ or $k_{L}$ is not possible owing to the fact that the final concentrations are not identical. The meaning of $J, \tau$, and $C_{0}$ are not the same in the single and multiple absorber simulations.

From Table II, we see that $k_{\nu_{A}}$ becomes independent of $D$ as $D$ gets large. This same behavior was noticed

TABLE II. Rate constants from single absorber and multiple absorber simulations as a function of diffusion constant.

\begin{tabular}{ccccc}
\hline \hline$D^{2}$ & \multicolumn{1}{c}{$C_{f}^{2}$} & $k_{M A}{ }^{2}$ & $k_{D^{2}}$ & $k_{L}{ }^{2}$ \\
\hline $1 \times 10^{-2}$ & $5.1 \times 10^{-3}$ & $1.33 \times 10^{-3}$ & $4.23 \times 10^{-2}$ & \\
$1 \times 10^{-3}$ & $5.2 \times 10^{-3}$ & $1.35 \times 10^{-3}$ & $5.87 \times 10^{-3}$ & \\
$5 \times 10^{-4}$ & $5.32 \times 10^{-3}$ & $1.27 \times 10^{-3}$ & $3.42 \times 10^{-3}$ & $7.11 \times 10^{-4}$ \\
$1 \times 10^{-4}$ & $5.78 \times 10^{-3}$ & $8.97 \times 10^{-4}$ & $1.05 \times 10^{-3}$ & $5.74 \times 10^{-4}$ \\
$5 \times 10^{-5}$ & $6.15 \times 10^{-3}$ & $6.86 \times 10^{-4}$ & $6.54 \times 10^{-4}$ & $4.94 \times 10^{-4}$ \\
$1 \times 10^{-5}$ & $6.90 \times 10^{-3}$ & $2.98 \times 10^{-4}$ & $2.33 \times 10^{-4}$ & \\
\hline
\end{tabular}

a.u. 
TABLE III. The diffusion equation ratio

[Eq. (19)] and multiple absorber

Langevin ratio of the Eley-Rideal to

Langmuir-Hinshelwood rates.

\begin{tabular}{ccc}
\hline \hline$D^{2}$ & $\delta_{\Sigma}$ & \multicolumn{1}{c}{$\delta$} \\
\hline $1 \times 10^{-2}$ & 0.37 & 0.012 \\
$1 \times 10^{-3}$ & 0.33 & 0.073 \\
$5 \times 10^{-4}$ & 0.36 & 0.12 \\
$1 \times 10^{-4}$ & 0.45 & 0.38 \\
$5 \times 10^{-5}$ & 0.54 & 0.61 \\
$1 \times 10^{-5}$ & 1.09 & 1.63 \\
\hline \hline
\end{tabular}

a.u.

in the single absorber Langevin simulations, and occurs because the particles do not exhibit any diffusive motion over distances on the order of the interparticle separations. As $D$ decreases, $k_{M A}$ exhibits a dependence on $D$, and when $D$ is on the order of $10^{-4}$ or $10^{-5}$ a. $u$. (the physical range of diffusion constants) the agreement between the rate constants is reasonably good. As the diffusion constant diminishes the rate constant decreases resulting in a higher final surface concentration.

The results presented in Table II are representative of the behavior of surface diffusion controlled reactions when $\tau$ is very short. We were unable to carry out simulations for large $\tau$ because the approach to steadystate behavior becomes prohibatively long for large $\tau$. The principal effect of making $\tau$ large is to increase $\gamma^{-1}$. In a true multiparticle surface reaction the concentration profile should reach an asymptote over a distance on the order of the interparticle separation. For the multiple absorber simulations reported here that distance is given by $C_{f}^{-1 / 2}$. For the diffusion constants used in this study, this distance ranges between 12 and 14 a. $u$. which is always larger than $\gamma^{-1}$. Consequently, $k_{M_{A}}$ and $k_{D}$ have been found to be in good agreement for physical values of the diffusion constant. However, for very large $\tau, \gamma^{-1}$ would become much larger than an interparticle spacing rendering the single absorber model inaccurate. A more accurate representation of the diffusion controlled rate constant would be a model with finite $R_{B}$ where $R_{B}$ is taken to be

$$
R_{B}=C_{f}^{-1 / 2} / 2 \text {. }
$$

For large $\tau$ [or small $\gamma$, see I, Eq. (55)] the finite $R_{B}$ rate constant reduces to $k_{D_{0}}$ [Eq. (8)].

The multiple absorber simulations also allowed us to calculate the ratio of the rate of Eley-Rideal reactions to the rate of Langmuir-Hinshelwood reactions. The Eley-Rideal rate was calculated by removing all reactant particles located within a distance $R_{A}$ of particles added by the external flux $J$. This calculated ratio is compared with the expression given in Eq. (79) of I,

$$
\delta(x)=\frac{x K_{0}(x)}{2 K_{1}(x)}
$$

where

$$
x=\gamma R_{A} \text {. }
$$

Equation (19) is based on the diffusion model. The multiple absorber Langevin values of $\delta, \delta_{L}$, are given in Table III along with results evaluated from Eq. (19). The agreement between the multiple absorber simulation ratio and the diffusion equation ratio is good for small values of $D$. As expected the agreement is poor for large $D$, because the diffusion equation becomes inaccurate for the reasons discussed previously.

\section{CONCLUSIONS}

In this work, we have carried out a critical analysis of some of the assumptions in our recent development of a two-dimensional isotropic model of diffusion influenced reactions on crystal surfaces. Using Langevin simulations we have found the single absorber model discussed in Sec. II to be well described by the diffusion equation for diffusion constants on the order of those expected for chemisorbed systems.

By analysis of multiabsorber Langevin simulations we have been able to infer that the single absorber model for surface reactions is inappropriate for strongly bound species with very long absorption lifetimes. For dilute weakly bound systems diffusion controlled rate constants can be best obtained from Eq. (7), whereas Eq. (8) is appropriate for strongly bound systems. When Eq. (8) is used $R_{B}$ should be taken from Eq. (18).

In I, we developed expressions for the activation energy and the ratio of the Eley-Rideal to LangmuirHinshelwood rates for diffusion controlled surface reactions at the infinite $R_{B}$ limit. If the finite $R_{B}$ limit is more appropriate the activation energy for the reaction is identical to the activation energy for diffusion in the diffusion controlled case [see Eq. (8)]. In the infinite $R_{B}$ case, the activation energy for diffusion controlled reactions is only approximately given by the activation energy for diffusion [see I, Eqs. (87) to (89)].

For long adsorption lifetimes when $R_{B}$ is finite and $\gamma$ approaches zero the ratio of the Eley-Rideal to Langmuir-Hinshelwood rates is given by (see I, Sec. III C for details of such developments)

$$
\begin{aligned}
& \delta=\frac{F_{A}}{F_{D}}, \\
& \delta=\frac{\pi R_{A^{J}}^{2}}{2 \pi D C_{0} / \ln \left(R_{B} / R_{A}\right)} .
\end{aligned}
$$

As shown in I for most physical systems, $C_{0}$ is the same order of magnitude as $J \tau$. When $C_{0}=J \tau, E q$. (22) becomes

$$
\delta=\frac{R_{A}^{2} \ln \left(R_{B} / R_{A}\right)}{2 \pi D \tau} .
$$

Since $\tau$ is large, $\delta$ will be small for cases, where $R_{B}$ is taken to be finite, and the Langmuir-Hinshelwood mechanism will dominate.

We are presently using the results developed in I and in the present work to extract rate information from calculated diffusion constants. The results of these calculations will appear separately. 


\section{ACKNOWLEDGMENTS}

One of us (DLF) wishes to thank the Chemistry Division of Los Alamos National Laboratory for its hospitality and support while on sabbatical leave from the University of Rhode Island. We wish to thank J. Winterkamp of Los Alamos National Laboratory's Group ESS-5 for computational support.

\section{APPENDIX}

In this Appendix, we derive the time dependent solution to Eq. (1) subject to the boundary conditions given in Eqs. (14) to (16). Our treatment parallels the discussion of heat flow in an infinite hollow cylinder given by Luikov. ${ }^{13}$ We also derive the short time behavior of the absorption rate in the infinite $R_{B}$ limit.

From Eq. (1), we write

$$
W(r, t)=W_{D}(r)+\rho(r, t),
$$

where $W_{D}(r)$ is the steady-state solution to Eq. (1) which satisfies

$$
D \nabla^{2} W_{D}(r)-\frac{W_{D}(r)}{\tau}+J=0,
$$

and $p(r, t)$ is the transient solution to Eq. (1) which satisfies

$$
\frac{\partial \rho}{\partial t}=D \nabla^{2} \rho-\frac{\rho}{\tau}
$$

The steady-state solution was derived in I and is given in Eq. (5).

The transient solution must satisfy the boundary conditions

$$
\begin{array}{ll}
\rho\left(R_{A}, t\right)=0, & t>0, \\
\rho\left(R_{B}, t\right)=0, & t>0,
\end{array}
$$

and

$$
\rho(r, 0)=C_{0}-W_{D}(r), \quad R_{A}<r \leq R_{B} .
$$

It is useful to write

$$
\rho(r, t)=f(r) \exp \left(-\xi^{2} D t\right) .
$$

If we introduce Eq. (A7) into Eq. (A3), we find that

$$
\nabla^{2} f+\alpha^{2} f=0,
$$

where

$$
\alpha^{2}=\xi^{2}-\gamma^{2} \text {. }
$$

Introducing the two-dimensional Laplacian into Eq. (A8), it is elementary to show that

$$
f=G J_{0}(\alpha r)+H Y_{0}(\alpha r),
$$

where $G$ and $H$ are coefficients to be determined by the boundary conditions, and $J_{n}(x)$ and $Y_{n}(x)$ are Bessel functions of the first and second kind of order $n$. Then,

$$
\rho(r, t)=\exp (-t / \tau)\left[G J_{0}(\alpha r)+H Y_{0}(\alpha r)\right] \exp \left(-\alpha^{2} D t\right) .
$$

Equations (A4) and (A5) imply that

$$
\begin{aligned}
& G J_{0}\left(\alpha R_{A}\right)+H Y_{0}\left(\alpha R_{A}\right)=0, \\
& G J_{0}\left(\alpha R_{B}\right)+H Y_{0}\left(\alpha R_{B}\right)=0 .
\end{aligned}
$$

Equations (A12) and (A13) have nontrivial solutions only when

$$
J_{0}\left(\alpha R_{A}\right) Y_{0}\left(\alpha R_{B}\right)-J_{0}\left(\alpha R_{B}\right) Y_{0}\left(\alpha R_{A}\right)=0 .
$$

Equation (A14) has an infinite number of real roots, ${ }^{13}$ $\alpha_{n}$ so that

$\rho(r, t)=\exp (-t / \tau) \sum_{n=1}^{\infty}\left[G_{n} J_{0}\left(\alpha_{n} \gamma\right)+H_{n} Y_{0}\left(\alpha_{n} r\right)\right] \exp \left(-\alpha_{n}^{2} D t\right)$

which can be rearranged to

$$
\rho(r, t)=\exp (-t / \tau) \sum_{n=1}^{\infty} c_{n} V_{0}\left(\alpha_{n} r\right) \exp \left(-\alpha_{n}^{2} D t\right),
$$

where

$V_{0}\left(\alpha_{n} r\right)=J_{0}\left(\alpha_{n} r\right) Y_{0}\left(\alpha_{n} R_{A}\right)-J_{0}\left(\alpha_{n} R_{A}\right) Y_{0}\left(\alpha_{n} r\right)$.

The coefficients $\left\{c_{n}\right\}$ are determined from Eq. (A6), or

$$
c_{0}-W_{D}(r)=\sum_{n=1}^{\infty} c_{n} V_{0}\left(\alpha_{n} r\right)
$$

Using orthogonality properties of $V_{0}\left(\alpha_{n} r\right),{ }^{13} \mathrm{Eq}$. (A18) can be solved to give the coefficients

$c_{n}=\frac{\pi^{2} \alpha_{n}^{2} J_{0}^{2}\left(\alpha_{n} R_{B}\right)}{2\left[J_{0}^{2}\left(\alpha_{n} R_{A}\right)-J_{0}^{2}\left(\alpha_{n} R_{B}\right)\right]} \int_{R_{A}}^{R_{B}} n\left[C_{0}-W_{D}(r)\right] V_{0}\left(\alpha_{n} r\right) d r$.

The integrals in Eq. (A19) can be evaluated from tables ${ }^{14}$ to give

$$
\begin{aligned}
c_{n}= & \frac{\pi J_{0}\left(m \mu_{n}\right)}{J_{0}\left(\mu_{n}\right)+J_{0}\left(m \mu_{n}\right)}\left[C_{0}-J \tau+\left[\left(\frac{\mu_{n}}{R_{a}}\right)^{2}-\gamma^{2}\right]^{-1}\right. \\
& \left.\times\left\{J \tau-C_{0} \frac{J_{0}\left(\mu_{n}\right)}{J_{0}\left(\mu_{n}\right)-J_{0}\left(m \mu_{n}\right)}\right\}\right],
\end{aligned}
$$

where

$$
\mu_{n}=\alpha_{n} R_{A}
$$

and

$$
m=R_{B} / R_{A} \text {. }
$$

With Eq. (A20), the derivation of Eq. (13) is complete.

It is useful to examine the behavior of the transient solution in a number of limits. From Eq. (A20), we immediately obtain

$$
\lim _{r \rightarrow 0} c_{n}=\frac{\pi J_{0}\left(m \mu_{n}\right) C_{0}}{J_{0}\left(\mu_{n}\right)+J_{0}\left(m \mu_{n}\right)}\left[1-\frac{J_{0}\left(\mu_{n}\right)}{J_{0}\left(\mu_{n}\right)-J_{0}\left(m \mu_{n}\right)}\right],
$$

which is the $n$th coefficient of the two-dimensional transient diffusion equation. Another interesting limit is that for infinite $R_{B}$. For simplicity, we evaluate this limit for $C_{0}=J \tau$. Using the relation

$$
\lim _{m \rightarrow \infty} \mu_{n}=\frac{n \pi}{m} \text {, }
$$

we have from $\mathrm{Eq}$. (A20),

$$
\lim _{R_{B^{-\infty}}} c_{n} \equiv c_{n^{\infty}}
$$

$$
=\frac{\pi J_{0}(n \pi) C_{0}}{J_{0}\left(\frac{n \pi}{m}\right)+J_{0}(n \pi)} \frac{\left(\frac{n \pi}{R_{B}}\right)^{2}}{\left(\frac{n \pi}{R_{B}}\right)^{2}-\gamma^{2}}\left[1-\frac{J_{0}\left(\frac{n \pi}{m}\right)}{J_{0}\left(\frac{n \pi}{m}\right)-J_{0}(n \pi)}\right] .
$$


Using

$$
\lim _{m \rightarrow \infty} J_{0}\left(\frac{n \pi}{m}\right)=1,
$$

Eq. (A26) becomes

$c_{n \infty}=-\pi C_{0} \lim _{R_{B} \rightarrow \infty} \frac{J_{0}^{2}(n \pi)}{1-J_{0}^{2}(n \pi)} \frac{\left(\frac{n \pi}{R_{B}}\right)^{2}}{\left(\frac{n \pi}{R_{B}}\right)^{2}-\gamma^{2}}$.

Using Eqs. (A16) and (A28) with the definition of the transient rate

$$
F_{t}=2 \pi R_{A} D\left(\frac{\partial \rho}{\partial r}\right)_{r=R_{A}},
$$

we obtain

$$
\begin{aligned}
& \lim _{R_{B \rightarrow \infty}} F_{t}=-4 \pi C_{0} D \exp (-t / \tau) \\
& \quad \times \lim _{R_{B \rightarrow \infty}} \sum_{n=1}^{\infty} \frac{J_{0}^{2}(n \pi)}{1-J_{0}^{2}(n \pi)} \frac{\left(\frac{n \pi}{R_{B}}\right)^{2}}{\left(\frac{n \pi}{R_{B}}\right)^{2}-\gamma^{2}} \exp \left(-n^{2} \pi^{2} D t / R_{B}^{2}\right) .
\end{aligned}
$$

At the infinite $R_{B}$ limit, we may approximate the summation in Eq. (A30) by an integration to obtain

$$
\begin{aligned}
\lim _{R_{B^{\rightarrow}}} F_{t} \cong & -\lim _{R_{B^{\rightarrow \infty}}} 4 R_{B} C_{0} D \exp (-t / \tau) \\
& \times \int_{r / R_{B}}^{\infty} d y \frac{J_{0}^{2}\left(y R_{B}\right)}{1-J_{0}^{2}\left(y R_{B}\right)} \frac{y^{2}}{y^{2}-\gamma^{2}} \exp \left(-y^{2} D t\right)
\end{aligned}
$$

If we replace the Bessel functions by their asymptotic forms

$$
\lim _{R_{B^{-\infty}}} J_{0}\left(y R_{B}\right)=\left[\frac{2}{\pi y R_{B}}\right]^{1 / 2} \cos \left(y R_{B}-\frac{\pi}{4}\right)
$$

and the $\cos ^{2}\left[y R_{B}-(\pi / 4)\right]$ terms by their average values Eq. (A31) can be evaluated to give

$$
\lim _{R_{B^{-\infty}}} F_{t} \cong-\frac{2 C_{0}}{\pi} D E_{i}\left(\frac{t}{\tau}\right)
$$

where $E_{i}(x)$ is the exponential integral function. ${ }^{15}$ At shor times this rate is divergent

$$
\lim _{t \rightarrow 0} \lim _{R_{B^{\rightarrow \infty}}} F_{t} \cong-\frac{2 C_{0}}{\pi} D \ln \frac{t}{\tau} \text {. }
$$

'J. D. Doll and H. K. McDowell, J. Chem. Phys. 77, 479 (1982).

${ }^{2}$ H. K. McDowell and J. D. Doll, Surf. Sci. 121, L537 (1982).

${ }^{3}$ J. D. Doll and H. K. McDowell, Surf. Sci. 123, 99 (1982).

${ }^{4} \mathrm{H} . \mathrm{K}$. McDowell and J. D. Doll, J. Chem. Phys. 78, 3219 (1983).

${ }^{5}$ G. Ehrlich and K. Stolt, Anmu. Rev. Phys. Chem. 31, 603 (1980).

${ }^{6}$ D. L. Freeman and J. D. Doll, J. Chem. Phys. 78, 6002 (1983).

${ }^{7}$ F. C. Collins and G. E. Kimball, J. Colloid Sci. 4, 425 (1949).

${ }^{8}$ M. J. Stowell, Philos. Mag. 26, 349 (1972).

${ }^{9}$ S. Chandrasekhar, Rev. Mod. Phys, 15, 1 (1943).

${ }^{10} \mathrm{~J}$. P. Boon and S. Yip, Molecular Hydrodynamics (McGrawHill, New York, 1980), Chap. 2.

${ }^{11} \mathrm{~S}$. A. Adleman, Adv. Chem. Phys. (in press).

${ }^{12}$ S. Harris, J. Chem. Phys. 75, 3103 (1981).

${ }^{13}$ A. V. Luikov, Analytical Heat Diffusion Theory (Academic, New York, 1968), Chap. 4.

${ }^{14}$ I. S. Gradshteyn and I. M. Ryzhik, Tables of Integrals, Series, and Products (Academic, New York, 1965).

${ }^{15} \mathrm{M}$. Abramowitz and I. A. Stegun, Handbook of Mathematical Functions (Natl. Bur. Stand. U.S., Spec. Publ., Washington, D.C., 1964), Chap. 5. 